\title{
Osteoarthritis in the forefoot accompanied with joint pain is potentially associated with bone alterations
}

\author{
Yukio Nakamura ${ }^{1}$, Mikio Kamimura ${ }^{2}$, Shigeharu Uchiyama ${ }^{1}$, Masatoshi Komatsu ${ }^{1}$, Shota \\ I kegami $^{1}$, Hiroyuki Kato ${ }^{1}$ \\ 1. Department of Orthopaedic Surgery, Shinshu University School of Medicine, Matsumoto, Japan. 2. Center of \\ Osteoporosis and Spinal Disorders, Kamimura Orthopaedic Clinic, Matsumoto, Japan.
}

Correspondence: Yukio Nakamura. Address: Department of Orthopaedic Surgery, Shinshu University School of Medicine, Asahi 3-1-1, Matsumoto 390-8621, Japan. Email: yxn14@aol.jp

Received: December 13, 2014

Accepted: March 30, 2015

Online Published: J une 3, 2015

DOI : $10.5430 /$ ijdi.v2n2p64

URL: http://dx.doi.org/10.5430/ijdi.v2n2p64

\section{Abstract}

We previously reported that the pathophysiology of hip Osteoarthritis (OA) may be due primarily to bone alterations. In the present case series, we describe the clinical results of 4 patients with forefoot OA and accompanying joint pain. Magnetic resonance imaging (MRI) and plain radiographs again revealed that bone alterations could have been the cause of the $\mathrm{OA}$ and pain.

\section{Keywords}

Small joints, Osteoarthritis, Bone alterations, Magnetic resonance imaging, Joint pain

\section{I ntroduction}

Osteoarthritis (OA) is the most common disease of the joints and affects more than $60 \%$ of the elderly population. The pathogenesis of primary OA suggests an intrinsic disease of the cartilage, whereby biochemical and metabolic alterations result in tissue breakdown ${ }^{[1]}$. Recently, OA has been considered to be a whole-joint disease process that involves multiple tissues in and around the afflicted joint ${ }^{[2,3]}$.

In a survey of Japanese elderly people aged 60 years and over, Muraki et al. reported that whereas $47.0 \%$ of men and $70.2 \%$ of women possessed radiographic knee OA, the incidence of knee pain was only $21.2 \%$ and $27.3 \%$, respectively ${ }^{[4]}$. Clegg et al. found that in patients with OA of the knee, joint pain had spontaneously improved 24 weeks after onset, even in a non-medicated placebo group ${ }^{[5]}$. These collective results indicate that there are numerous OA patients who do not experience joint pain, although the precise mechanism by which pain occurs is not well understood.

Modern imaging modalities are playing an increasingly important role in the assessment of structural OA progression and identification of the underlying disease processes. Although radiography has conventionally been employed to these ends, OA symptoms are often not associated with the structural abnormalities depicted by radiographs. Such dissociation between patient complaint and outcome measure has underscored the need for other established modalities of OA evaluation, such as magnetic resonance imaging (MRI). Semi-quantitative whole-organ assessment of OA has recently 
been achieved using MRI and represents a promising advance in disease prognosis ${ }^{[3]}$. Nevertheless, it remains challenging to elucidate the associations between $\mathrm{OA}$ and joint pain.

We routinely evaluate OA status using both radiography and MRI at our institutions. Based on our observations, we earlier proposed that the major pathophysiology of hip, shoulder, and knee OA with accompanying joint pain may be due to bone alterations ${ }^{[6-9]}$. While we often encounter patients without serious radiographic OA complaining of joint pain, even in the small joints, there are also numerous cases of advanced radiographic OA with no pain in the affected bone regions. As far as we know, there have been no MRI-based studies to date on OA or joint pain progression in the small joints.

The present case series describes the MRI and radiographic findings of several patients with painful OA in the forefoot and addresses the pathophysiology of OA with accompanying pain as measured by the Denis pain scale ${ }^{[10]}$.

\section{Patients and methods}

The patients recruited in this series complained of strong or markedly worsened joint pain at presentation. Plain radiographs were taken at the first visit, and MRI was performed on all affected joints within a month afterwards. Conservative treatment was advised for all patients. In total, we examined the clinical data of 2 Lisfranc joints and 2 hallux cuneiform metatarsal joints (CMJ) of 2 primary OA patients each.

Pain was assessed using the pain scale reported by Denis, ${ }^{[10]}$ as follows: P1, no pain; P2, occasional minimum pain with no need for medication; P3, moderate pain with occasional medication but no interruption of work or significant changes in activities of daily living (ADL); P4, moderate to severe pain with frequent medication and occasional absence from work or significant change in ADL; and P5, constant or severe incapacitating pain requiring chronic medication. Primary OA was classified as Kellgren-Lawrence (KL) grading I to IV ${ }^{[11]}$.

The MRI acquisition conditions in this study were the following: coronal and sagittal views using T1-weighted imaging (T1W), TR: 530 (SI), FA: 90 (SI), and TE: 14 (SI); and short $\tau$ inversion recovery (STIR) for skeletal survey, TR: 5010 (SI), FA: 180 (SI), TE: 71 (SI), and TI: 150 (SI). The strength of the magnetic field was $1.5 \mathrm{~T}$ (SI). Bone signal changes were judged to be present only when alterations were detected by both T1W and STIR.

All patients were instructed to take non-steroidal anti-inflammatory drugs and/or tramadol when necessary for joint pain. The subjects were in good general health and did not have any history of serious illnesses or complications. The present study was approved by all institutional ethics committees. Informed written consent was obtained from each patient.

\section{Results}

All 4 patients had P3 pain at presentation according to the Denis pain scale and initially exhibited low-intensity signals in T1W images and high-intensity signals in STIR images in afflicted bone regions. Conservative treatment remarkably improved forefoot pain in all cases.

\subsection{Case 1: 53-year-old woman with left Lisfranc joint OA}

Left forefoot pain had appeared suddenly 2 weeks prior to presentation. Swelling and tenderness were noted around the left second Lisfranc joint. Plain radiographs showed mild OA of KL grade II. The pain had disappeared (P1) 2 months after onset and she was able to resume her hobby of dancing. Lumbar (L1-4) bone mineral density (BMD) was 1.013 (T-score: -0.8) and total hip BMD was 0.872 (T-score: -0.5) (see Figure 1). 


\subsection{Case 2: 51-year-old woman with Lisfranc joint OA}

Left forefoot pain had manifested suddenly 5 months before presentation. Tenderness was noted along the left first metatarsal bone. Plain radiographs showed mild OA of KL grade II (data not shown). MRI revealed bone signal changes in the first Lisfranc joint. One month later, the pain had disappeared (P1).
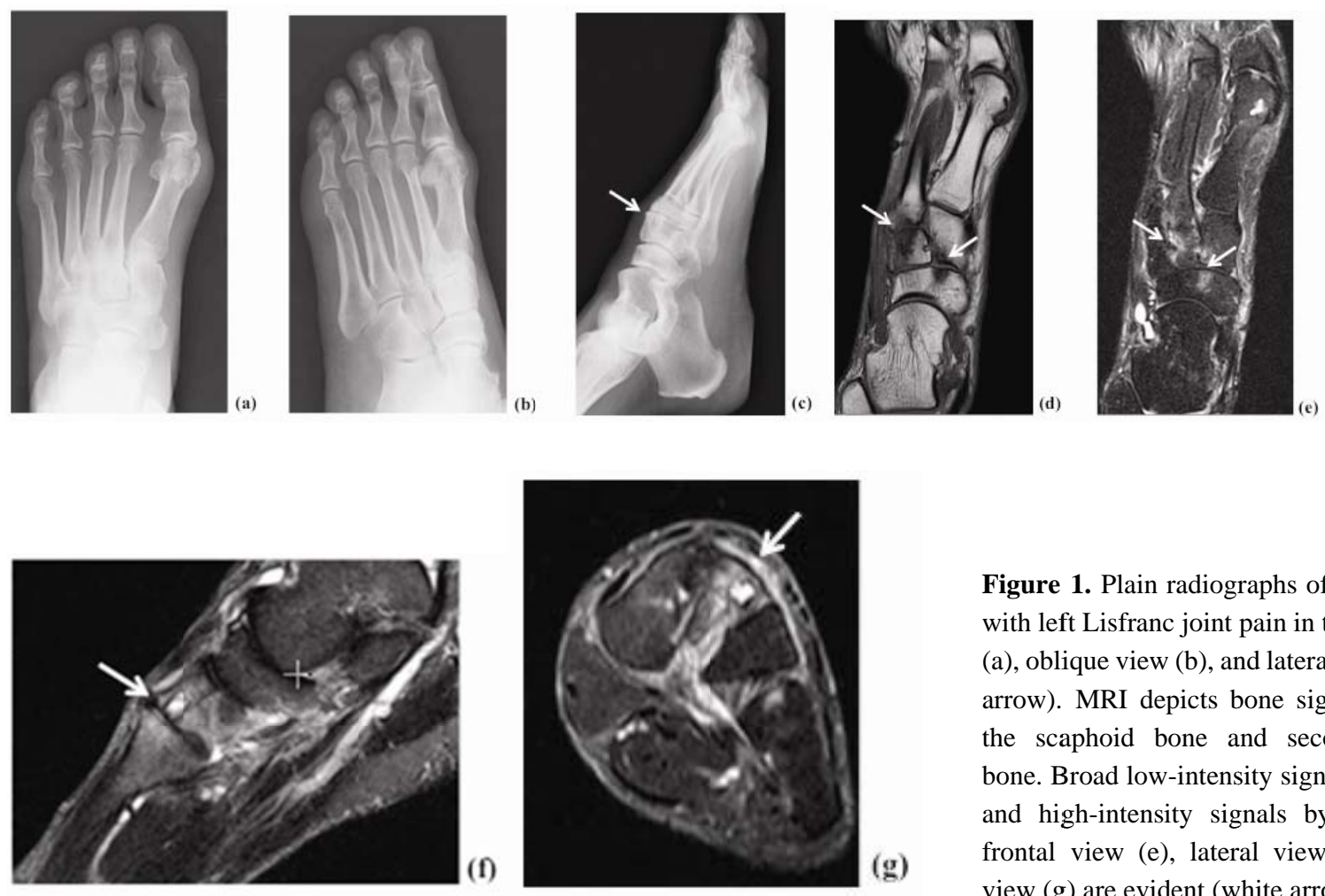

(g)

Figure 1. Plain radiographs of an OA patient with left Lisfranc joint pain in the frontal view (a), oblique view (b), and lateral view (c, white arrow). MRI depicts bone signal changes in the scaphoid bone and second metatarsal bone. Broad low-intensity signals by T1W (d) and high-intensity signals by STIR in the frontal view (e), lateral view (f), and axial view (g) are evident (white arrows).

\subsection{Case 3: 76-year-old man with hallux CMJ OA}

Right first toe pain had appeared without episode 1 month before seeking medical attention, at which time plain radiographs showed obvious joint space narrowing but no hallux valgus (HV) and KL grade III. MRI examination uncovered bone signal changes in the hallux CMJ. The joint pain was undetectable (P1) at 2 months after presentation (see Figure 2).

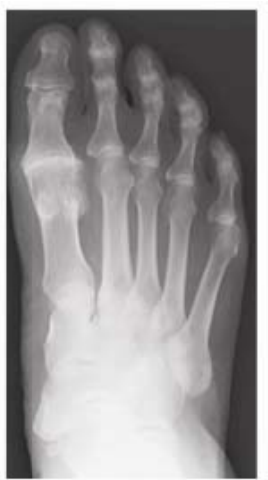

(a)

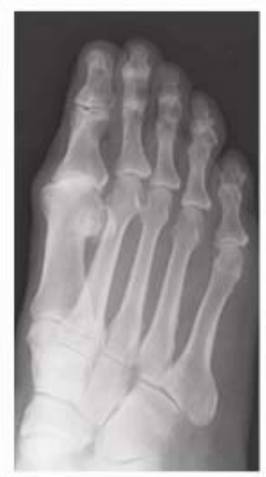

(b)

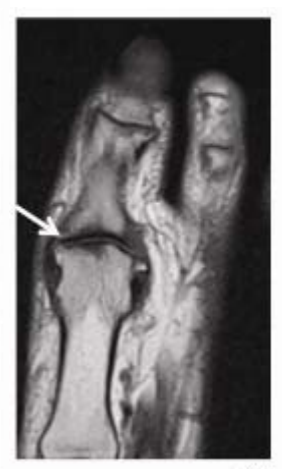

(c)

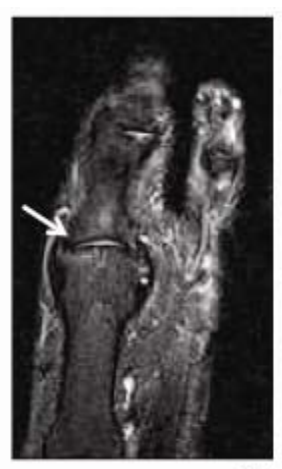

(d)

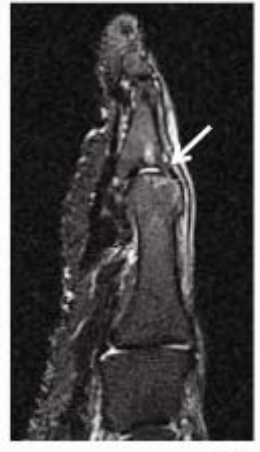

(e)

Figure 2. Plain radiographs of an OA patient with left CMJ pain in the frontal view (a) and oblique view (b) One month after pain onset, MRI demonstrates bone signal changes in the left first CMJ. Broad low-intensity signals by T1W (c) and high-intensity signals by STIR in the frontal view (d) and lateral view (e) are observed (white arrows). 


\subsection{Case 4: 64-year-old woman with hallux CMJ OA}

Left forefoot pain had improved and then worsened 3 years prior to presentation. The pain had again increased considerably 1 month before revisiting our institution. Plain radiographs showed mild OA with accompanying HV and KL grade II (data not shown). MRI disclosed bone signal changes in the left hallux CMJ. The use of a foot plate ameliorated her joint pain. L1-4 BMD was 0.939 (T-score: -1.5) and total hip BMD was 0.768 (T-score: -1.4).

\section{Discussion}

The frequency of OA in weight-bearing joints, such as the hip and knee, is considerably higher than that in the ankle. The incidence of small joint OA is also low ${ }^{[12]}$, and it remains uncertain how joint pain and OA progression occur in the small joints.

There are few reports on Lisfranc joint OA and its exact prevalence is currently unknown. This condition is typically observed in middle-aged and elderly individuals. The Lisfranc joint is comprised of 6 articulations, the weak dorsal ligaments, and the strong plantar ligaments. The Lisfranc ligament serves to secure the second metatarsal in the keystone of the midfoot. Traumatic ligament injury and fracture can result in deformity, instability, pain, and degenerative disease of the Lisfranc joint ${ }^{[13]}$. However, most patients with Lisfranc OA do not have joint pain or a history of trauma. In Japan, Lisfranc OA frequently disrupts the ability to sit straight due to an OA-accompanying hump, but pain is uncommon ${ }^{[13]}$.

Pain occurring in first MTP joint OA has been associated with reduced general and foot-specific health-related quality of life $^{[14,15]}$. First MTP joint OA is characterized by localized symptoms of pain and stiffness in the affected region ${ }^{[16]}$. HV is a highly prevalent forefoot deformity ${ }^{[17]}$ presenting as lateral deviation of the first toe and progressive subluxation of the first MTP joint. HV is often associated with first MTP joint OA ${ }^{[18]}$. The prevalence of first MTP joint OA is not fully known. However, population-based studies indicate that its incidence increases with age in the general population and that its overall frequency is as high as $35 \%$ among individuals between 35 and 60 years of age ${ }^{[19]}$.

The above reports confirm that the pathophysiology of OA and the cause of joint pain are unclear in forefoot joint OA. Although MRI may contribute to the improved clinical care of patients with forefoot OA, there appear to be no reports addressing OA progression, joint pain, and MRI findings in this condition.

The present series revealed that bone alterations in patients with OA and accompanying joint pain, even in small joints, were depicted as low-intensity signals by T1W and high-intensity signals by STIR. Conservative treatment resolved the pain in all cases.

There have been several accounts of the cause of joint pain in OA being attributed to joint findings in MRI. Guermazi et al. reviewed that MRI bone signal changes in joints sometimes suggested microfracture ${ }^{[20]}$. We previously reported: 1) the presence of bone signal changes detected by MRI in hip OA patients with accompanying joint pain, 2) the absence of bone signal changes in MRI of patients with hip OA, even in advanced cases, with no accompanying joint pain, and 3) that the main cause of joint pain could possibly be due to bone alterations ${ }^{[6,7]}$. Furthermore, we have described that knee and shoulder OA may be associated with joint pain and MRI-detectable bone alterations ${ }^{[8,9]}$.

In this study, bone alterations depicted by MRI were present in all patients with painful small joint OA. However, we witnessed that the pain resolved spontaneously in all cases. These findings suggest that some cases of pain associated with small joint $\mathrm{OA}$ are caused not by cartilage degeneration, but rather by bone alterations.

While low-intensity signals in T1W and high-intensity signals in T2W/STIR images generally indicate bone marrow edema ${ }^{[21]}$, these findings are not specific to bone fractures. Taljanovic et al. concluded that the amount of bone marrow edema in the OA hip as evaluated by MRI correlated with pain severity, radiographic findings, and microfractures ${ }^{\text {[22] }}$. 
Guermazi et al. have also recently shown that such MRI signal changes in joints potentially suggest microfractures ${ }^{[20]}$. Based on the results of our previous studies ${ }^{[6-9]}$ and others ${ }^{[20]}$, we consider that bone signal changes detected by MRI may be representative of microfractures, which in turn cause joint pain. In support of this, patients with hip OA and continuous joint pain showed persistent bone signal changes in MRI, while those in whom the pain had disappeared exhibited an absence of signal changes ${ }^{[23]}$. Similar findings have been described for knee OA ${ }^{[9]}$. Accordingly, we suspect that MRI bone signal changes also disappear over time along with the resolution of joint pain in forefoot OA.

The main limitation of this study is a short observation period that precluded end-point radiographic and MRI examinations to confirm the absence of bone alterations in MRI corresponding to the disappearance of pain since the subjects ceased visiting our institution when their pain subsided. Further long-term studies of larger cohorts are needed to confirm our results.

\section{Conclusion}

MRI bone alterations were evident in painful forefoot OA joints. This pain resolved spontaneously within several months of conservative treatment. There were no obvious bone alterations detected by MRI in the surrounding non-painful joints despite also being afflicted with OA. Our findings suggest a relationship between MRI findings and joint pain, even in the case of small joint OA.

\section{Acknowledgments}

We thank radiologists Dr. Hiroshi Koshihara and Dr. Tomoko Furukawa at Chushin Matsumoto Hospital for their invaluable help.

\section{References}

[1] Moskowitz RW, Howell DS, Altman RD, et al: Osteoarthritis, 3ed. Diagnosis and Medical/Surgical Management, Philadelphia: W.B. Saunders, 2003.

[2] Guermazi A, Niu J, Hayashi D, et al. Prevalence of abnormalities in knees detected by MRI in adults without knee osteoarthritis: population based observational study (Framingham Osteoarthritis Study). BMJ. 2012; 345: e5339. PMid:22932918 http://dx.doi.org/10.1136/bmj.e5339

[3] Roemer FW, Guermazi A. MR imaging-based semiquantitative assessment in osteoarthritis. Radiol Clin North Am. 2009; 47: 633-54. PMid:19631073 http://dx.doi.org/10.1016/j.rcl.2009.03.005

[4] Muraki S, Akune T, Oka H, et al. Incidence and risk factors for radiographic knee osteoarthritis and knee pain in Japanese men and women: a longitudinal population-based cohort study. Arthritis Rheum. 2012; 64: 1447-56. PMid:22135156 http://dx.doi.org/10.1002/art.33508

[5] Clegg DO, Reda DJ, Harris CL, et al. Glucosamine, chondroitin sulfate, and the two in combination for painful knee osteoarthritis. N Engl J Med. 2006; 354: 795-808. PMid:16495392 http://dx.doi.org/10.1056/NEJMoa052771

[6] Kamimura M, Nakamura Y, Ikegami S, et al. Joint pain undergoes a transition in accordance with signal changes of bones detected by MRI in hip osteoarthritis. Open Rheumatol J. 2013; 7: 58-65. PMid:24133552 http://dx.doi.org/10.2174/1874312920130823002

[7] Kamimura M, Nakamura Y, Ikegami S, et al. The pathophysiology of primary hip osteoarthritis may originate from bone alterations. Open Rheumatol J Open Rheumatol J. 2013; 7: 112-118. PMid:24358070 http://dx.doi.org/10.2174/1874312920130930003

[8] Komatsu M, Nakamura Y, Kamimura M, et al. Bone alternations in painful osteoarthritic shoulder. J Case Reports. $2015:$ 84-87.

[9] Komatsu M, Nakamura Y, Kamimura M, et al. Knee Joint Pain Potentially Due to Bone Alterations in a Knee Osteoarthritis Patient. Am J Case Rep. 2014; 15: 534-7. PMid:25436838 http://dx.doi.org/10.12659/AJCR.891233

[10] Denis F, Armstrong GW, Searls K, et al. Acute thoracolumbar burst fractures in the absence of neurologic deficit. A comparison between operative and nonoperative treatment. Clin Orthop Relat Res. 1984; 189: 142-9.

http://dx.doi.org/10.1097/00003086-198410000-00015 
[11] Kellgren JH, Lawrence JS. Radiological assessment of osteo-arthrosis. Ann Rheum Dis. 1957; 16: 494-502. http://dx.doi.org/10.1136/ard.16.4.494

[12] Haugen IK, Hammer HB. Role of Modern Imaging Techniques in Hand Osteoarthritis Research and Clinical Practice. Curr Rheumatol Rep. 2014; 16: 399. PMid:24357444 http://dx.doi.org/10.1007/s11926-013-0399-y

[13] Chaney DM. The Lisfranc joint. Clin Podiatr Med Surg. 2010; 27: 547-60. PMid:20934104 http://dx.doi.org/10.1016/j.cpm.2010.06.005

[14] Abhishek A, Roddy E, Zhang W, et al. Are hallux valgus and big toe pain associated with impaired quality of life? A cross-sectional study. Osteoarthritis Cartilage. 2010; 18: 923-6. PMid:20417286 http://dx.doi.org/10.1016/j.joca.2010.03.011

[15] Gilheany MF, Landorf KB, Robinson P. Hallux valgus and hallux rigidus: a comparison of impact on health-related quality of life in patients presenting to foot surgeons in Australia. J Foot Ankle Res. 2008; 1: 14. PMid:19077213 http://dx.doi.org/10.1186/1757-1146-1-14

[16] Camasta C. Hallux limitus and hallux rigidus. Clin Pod Med Surg. 1996; 13: 425-8.

[17] Nix S, Smith M, Vicenzino B. Prevalence of hallux valgus in the general population: a systematic review and meta-analysis. J Foot Ankle Res. 2010; 3: 21. PMid:20868524 http://dx.doi.org/10.1186/1757-1146-3-21

[18] D'Arcangelo P, Landorf KB, Munteanu SE, et al. Radiographic correlates of hallux valgus severity in older people. J Foot Ankle Res. 2010; 3: 20. PMid:20846367 http://dx.doi.org/10.1186/1757-1146-3-20

[19] Trivedi B, Marshall M, Belcher J, et al. A systematic review of radiographic definitions of foot osteoarthritis in population-based studies. Osteoarthritis Cartilage. 2010; 18: 1027-35. PMid:20472083 http://dx.doi.org/10.1016/j.joca.2010.05.005

[20] Guermazi A, Roemer FW, Haugen IK, et al. MRI-based semiquantitative scoring of joint pathology in osteoarthritis. Nat Rev Rheumatol. 2013; 9: 236-251. PMid:23321609 http://dx.doi.org/10.1038/nrrheum.2012.223

[21] Koff MF, Potter HG. Noncontrast MR techniques and imaging of cartilage. Radiol Clin North Am. 2009; 47: $495-504$. PMid:19361672 http://dx.doi.org/10.1016/j.rcl.2009.01.004

[22] Taljanovic MS, Graham AR, Benjamin JB, et al. Bone marrow edema pattern in advanced hip osteoarthritis: quantitative assessment with magnetic resonance imaging and correlation with clinical examination, radiographic findings, and histopathology. Skeletal Radiol. 2008; 37: 423-31. PMid:18274742 http://dx.doi.org/10.1007/s00256-008-0446-3

[23] Kamimura M, Nakamura Y, Uchiyama S, et al. The pathophysiology and progression of hip osteoarthritis accompanying with joint pain are potentially due to primary bone alterations -Follow up study of hip OA patients. Open Rheumatol J. 2014; 8: 46-53. 